\title{
Quality and impact of Indian Doppler weather radar wind profiles: A diagnostic study
}

\author{
A. Sandeep, V. S. Prasad, C. J. Johny \\ National Centre for Medium Range Weather Forecasting, NOIDA - 201 309, India.
}

\begin{abstract}
:
In the tropics, improvement in weather forecasts requires good quality of vertical profiles of winds due to improper coupling of mass and wind fields and balance relationships in the region. Indian Meteorological Department (IMD) operates the network of Doppler Weather Radar (DWR) in microwave frequencies (S-band or C-band) at different locations in India. National Centre for Medium Range Weather Forecasting (NCMRWF) receives the Volume
\end{abstract} Velocity Processing (VVP) wind profiles from all operated DWR's via Global Telecommunication System (GTS) network in near real time. The radar VVP wind is a mean horizontal wind derived at different heights from radial velocity suitable for Numerical Weather Prediction (NWP) applications. Three numerical experiments CNTL (without VVP winds), 3DVAR and HYBRID with the assimilation of VVP winds using 3-dimensional variational (3dvar) and hybrid data assimilation systems were conducted using the NCMRWF Global Forecast System (NGFS) model. The objective of the paper is divided into two parts: 1) Quality of VVP winds 2) Impact of VVP wind profiles on NGFS model forecast. The quality of VVP wind profiles are assessed against the NGFS model background and radiosonde wind profiles. The absolute value of zonal and meridional wind observation minus background $(\mathrm{O}-\mathrm{B})$ is increasing with respect to the pressure for all DWR's. All radars are showing the accepted (rejected) ratio is decreasing (increasing) as a function of pressure. The resemblance of the zonal and meridional O-B statistics for 3DVAR and HYBRID experiments is apparently striking. A very good correspondence is seen between the accepted VVP winds and radiosonde winds in both experiments (3DVAR and HYBRID). The correlation coefficient $(\mathrm{R})$ is found to be higher at Patna (Patiala) for zonal (meridional) winds in 3DVAR experiment and at Patna (Jaipur) in HYBRID experiment, respectively. At Chennai, $\mathrm{R}$ value is found to be lower in both experiments for both wind components. On the other side, due to the assimilation of VVP winds using 3dvar, the root-mean-square-error (RMSE) of zonal and meridional winds are improved by $2-3 \%$ up to day 5 forecast with respect to the analysis below $700 \mathrm{hPa}$. Further improvement in RMSE ( 5\%) is found in both the wind components due to the hybrid data assimilation. The zonal and meridional wind RMSE of HYBRID (3DVAR) experiment is improved by $~ 5$ (3) \% with respect to 3DVAR (CNTL) experiment, respectively. 
